\title{
Adherencia in Vitro de Escherichia Coli 0111 a Células HEp-2
}

\author{
T.M. Guillemo Figueroa C.1; T.A. M. del Pilar Alcayde L.1; T.A. Vivian Soto G.1; \\ T.M. Orietta Ross K.2 \\ In Vitro Adherence to HEp-2 Cells and Plasmid \\ DNA Content of Escherichia Coli
}

\begin{abstract}
The ability to adhere to cultured HEp-2 cells and their plasmid DNA content was evaluated in 33 enteropathogenic Escherichia coli 0111 strains, 25 isolates cume from infants with diarthed and 8 from asymptomatic infants. Fourteen non pathogenje li. coli strains, considered to be part of the normal flora, were used as controls. No differences in the adhesive capacity of E. coli 0111 strains isolated from diarrhoeal cases (60\% or from asymptomatic subjects $(50 \%$ were detected. In contrast both groups of strains showed significant]y higher ( $p<0.001$ Fisher exact test). adherence rates when compared with strains from the control group (7\%). Plasmids with molecular weights 50 to 70 Md were detected in all strains investigated, irrespectively of their ability to adjere to $\mathrm{HEp}-2$ cells. HFp-2 assay constitutes a valuable disgnostic tool, that will probably improve our knowledge in the ethiology of dianheal diseases.

(Key' words: Escherichia coli Adherence to HEp2 cells. In vitro adherence. DNA plasmid content).
\end{abstract}

Numerosos estudios epidemiológicos han demostrado la asociación de infecciones con cierias cepas de Escherichia coli (E. coli o E.C.) y diarrea infantill,2. Más recientemente se han podido vislumbrar algunos mecanismos patogenicos implicados en la aparición de estos ca. $\operatorname{sos}^{3,4}$.

Hoy se reconocen al menos 4 clases de E. coli asociadas a afecciones entéricas en el hombre ${ }^{5}$; algunas cepas colonizan y liberan enterotoxinas (EC enterotóxica o ECET) en el intestino delga$\mathrm{do}^{6,7}$; otras (EC enteroinvasora o ECEJ) invaden e] colon originando síndromes disentéricos ${ }^{8}$; una tercera clase corresponde a los llamados serotipos enteropatogénicos (EC enteropatogénico 0 ECEP), los primeros en ser descritos, cuya patogenia aún no se conoce bien, y un cuarto tipo que produce una citotoxina semejante a la de las Shigellae ${ }^{9}$, como ha sjdo demostrado en el serotipo 0157:H7, en relación con colitis hemorrágica ${ }^{10,11}$.

De los grupos antes mencionados el que ha producido más controversias, por las dificultades para caracterizar las bases de su virulencia, es sin duda el de los denominados serotipos "clásicos" o ECEP: durañte la mayor parte de la década del 70 estos microorganismos fueron considerados

1. Unidad de Microbiologia, Instituto de Nutrición y Tecnología de los Alimentos. Universidad de Chile.

2. Laboratorio de Cultivos Celulares, Instituto de Salud Pública de Chile. no patógenos, incluso en muchos lugares se descontinuó su investigación. Sólo en 1978 a través de estudios en voluntarios se logró demostrar su indudable rol patogénico ${ }^{12}$; contemporáneamente se comprobó que la virulencia radicaba en la capacidad de adherencia ${ }^{13}$, aparentemente no mediada por fimbras ${ }^{14}$ : que inducía alteraciones histológiçus caracteristicas ("pedestal") en el epitelio intestinal colonizado, humano's y ani$\mathrm{mal}^{16,17}$

Los primeros ensayos in vitro para caracterizar este mecanismo patogénico, propio de algunos serotipos de ECEP, fueron desarrollados por Cravioto ${ }^{33}$, quién demostró que alrededor de $80 \%$ de las cepas enteropatogénicas (ECEP) adherian a células HEp--2. Este autor describrió además que la adherencia "in vitro" exa resistente a la manosa, más tarde $\mathrm{McNeish}{ }^{18}$ comprobó que el mecanismo de adherencia ocurría también con células del intestino fetal humano. Posteriormente Scaletsky ${ }^{19}$, asoció la virulencia con el fenómeno que llamó "adherencia localizada", propio de algunas cepas de ECEP, hallazgos que fueron confirmados "in vitro" usando células HEp-2 y $\mathrm{Hela}^{20}$.

Se ha demostrado también que la capacidad de adherencia de las ECEP parece estar genéticamente controlada por plasmidios cuyos pesos moleculares (PM) varian entre 50 y 70 megadalton $(\mathrm{Md})^{21}$, tal conocimiento se ha aplicado en la creación de noléculas que sirven de tientos o instrumentos de exploración ("probes") para el 
diagnóstico de estos serotipos enteropatogéni$\cos ^{2} 2$

La información epidemiológica sobre el fenómeno de adherencia de ECEP, es aún limitada, sin embargo ha permitido postular que los deno. minados serotipos "clásicos" pueden ser divididos en dos clases: los que desarrollan adherencia localizada, entre los que se incluyen los serotipos $055,0111,0119,0127,0128$ y $0142, y$, por otra parte, los que no expresan dicha capacidad, serogrupos 044, 086 y 0114 , de los que se desconoce el mecanismo de virulencia 22

Puesto que en Chile ECEP es el grupo bacteriano más frecuentemente relacionado con casos de diarrea infantil23.24 nos propusimos buscar "in vitro" la capacidad de adherencia de cepas de E. coli 0111 , obtenidas de niños con diarrea $y$ asintomáticos y comparándolas con la de cepas de $E$. coli pertenecientes a la flora normal. Además estudiamos la presencia de DNA plasmidial en dichas cepas, para ver si existen relaciones entre ésta y el fenómeno de adherencia.

\section{MATERIAL y METODO}

Se estudiaron 47 cepas de E. coli aisladas de deposiciones de niños, 33 correspondian al serogrupo 0111 (25 cepas provenían de niños con diatrea y 8 de niños asintomáticos. La identidad serológica de las cepas fue confirmada con antisueros comerciales Difco. Sólo las cepas que en el estudio de aglutinación en tubo, dieron título $\geqslant 1: 320$ fueron consideradas en el estudio.

Catorce cepas de E, coli de la flora normal fueron utilizadas como controles, comprobándose previamente que no producían toxinas, no eran invasoras (Séreny), ni pertenecían a algún serotipo enteropatógeno clásico.

\section{Ensayos de adherencia a células $H E p-2$ :}

Para este ensayo "in vitro" se usó, con algunas modificaciones, el método propuesto por Cravioto 13 . Se consideró que habia adherencia localtzada sólo cuando, mediante observación microscópica $(100 \mathrm{x})$, más de $40 \%$ de las células HEp--2 tenían microcolonias localizadas. Las modificaciones de la técrica consistieron en: uso de tampón fosfato salino $(\mathrm{pH}=7,2$ ) en el lavado de las células; disminución del tiempo de incubación a 3 horas; fijación de las células durante 15 minutos en metanol y aumento de la concentración del colorante Giemsa, para la tinción, al doble (20\%).

\section{Determinación de plasmidios:}

EI DNA piasmidial fue investigado produciendo lisis de tas cepas con dodecil sulfato de sodio y separándolo por electroforesis en geles de agarosa al $0,7 \%$ según la técnica descrita por Kado y Liu25, las corridas se realizaron a $50 \mathrm{~V} y$ durante 4 horas, $y$ los geles se fotografiaron bajo luz UV con cámara Polaroid tipo 55 El tamaño de los plasmidios se calculó extrapolanda en la recta obtenida con los controles de peso molecular, según lo propuesto por Meyers 26 . Los controdes incluyeron los plasmidios Plac (101 Md); R] (61 Md); RP4 (36 Md) y SA (26 Md).

\section{RESULTADOS}

La cantidad de cepas de E. coli 0111 que mostraban capacidad de adherencia localizada (Fig. 1) o difusa (Fig. 2) no fueron diferentes en las cepas que provenían de casos de diarrea $(60 \%)$

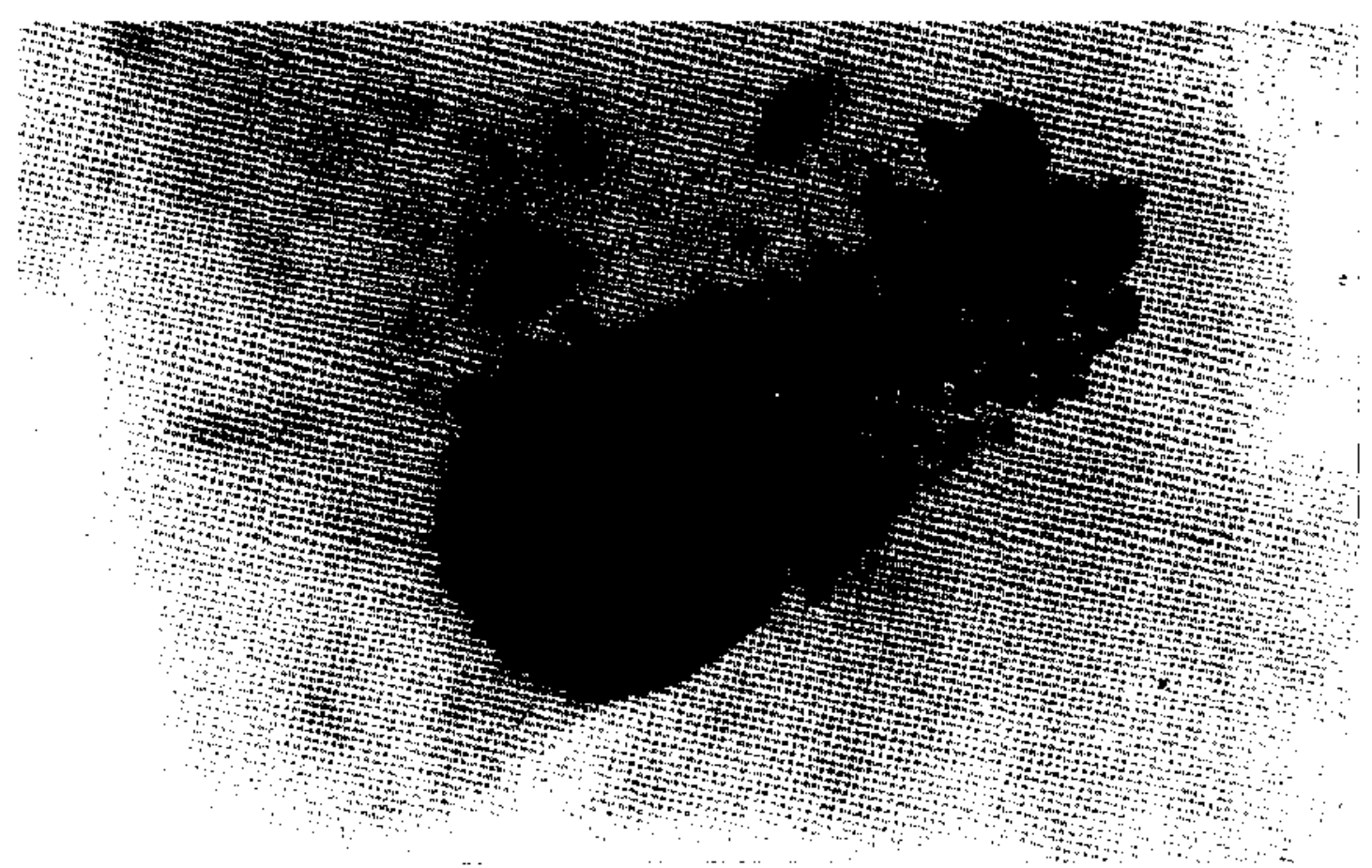

Fig. 1: Adherencia localizada en células $\mathbf{H E}_{\mathrm{P}}-2$. de una cepa de Escherichia coli 0111. 
- en aquellas de niños asintomáticos (50\%) (Tabla 1). Al comparar los resultados obtenidos en cualquiera de estos grupos con los provenientes de las cepas de E. coli pertenecientes a la flora normal, se hacen aparentes diferencias que son estadísticamente significativas ( $p<0,001$ prueba exacta de Fisher).

La identificación de plasmidios de $50 \mathrm{y}$ $70 \mathrm{Md}$, compatibles con los asociados a la capa- cidad de adherencia, no guardó relación con la presencia o ausencia de adherencia localizada ni con el hecho de provenir las cepas de casos de diarrea o de sujetos asintomáticos. Asimismo, la frecuencia con que fueron detectados plasmidios de estos rangos de PM no mostró diferencias entre cepas enteropatógenas y de flora normal (Tabla 2).

La Fig. 3 muestra los resultados de la electro-

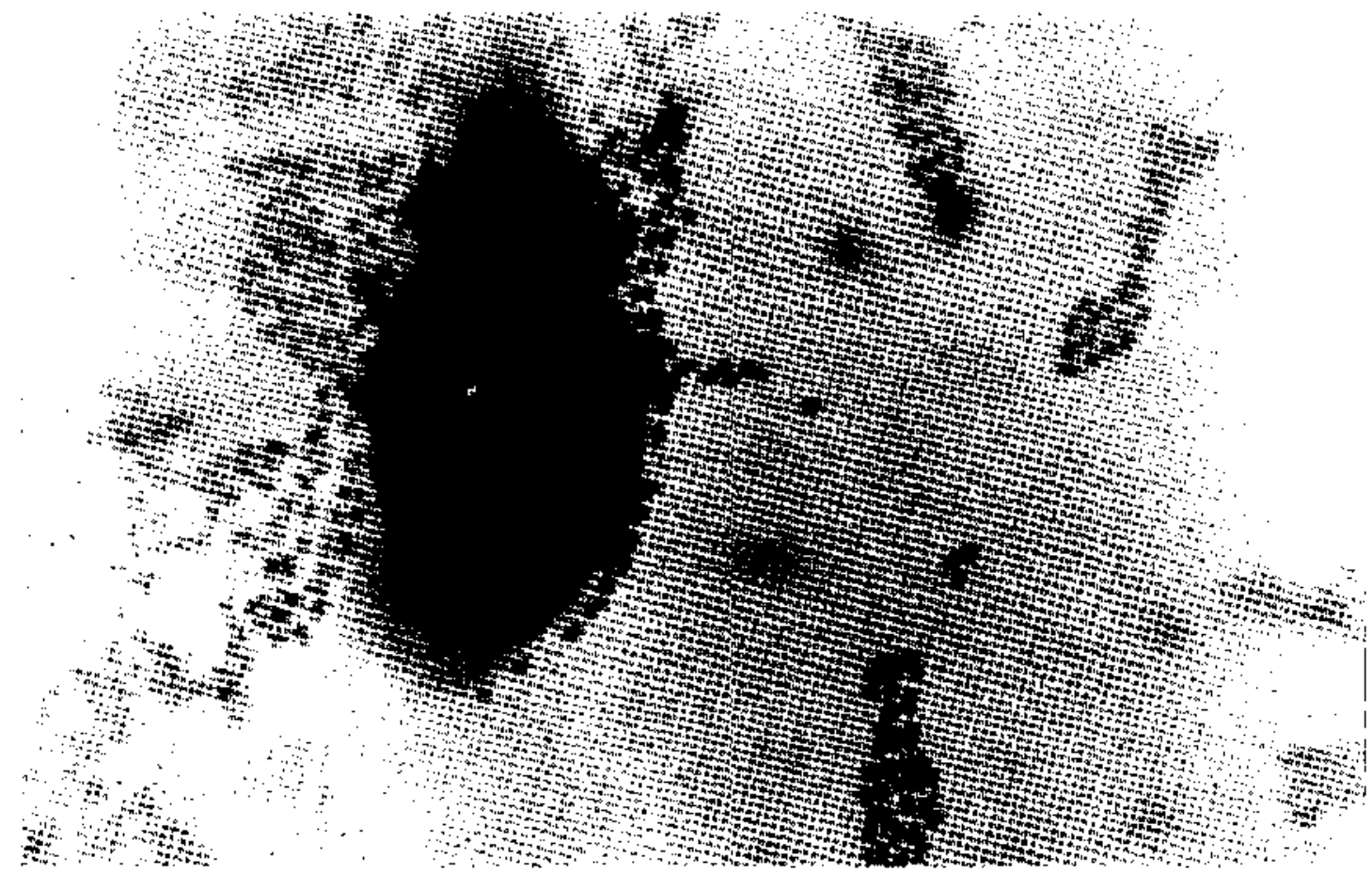

Fig. 2: Adharencia difusa en células HEp-2. de una cepa de Escher ichia coli 0111.

Tabla 1.

Distribución de Cepas de E. coli 0111 y de Flora Normal según su capacidad de Adherencia Localizada a Células $\mathrm{HEp}-2$

ADHERENCIA LOKALIZADA

\begin{tabular}{lcccc}
\hline & \multicolumn{2}{c}{ Positiva } & \multicolumn{2}{c}{ Negativa } \\
\hline E. coli 0111 & Diarreas & Asintomáticos & Diarreas & A sintomáticos \\
E. coli no & $15 / 25$ & $4 / 8$ & $10 / 25$ & $4 / 8$ \\
&,--- & $1 / 14$ &,---- & $13 / 14$
\end{tabular}

Enteropatógeno

Tabla 2.

Presencia de Plasmidios de entre 50-70 Md en las Cepas de E. coli 0111 y de Flora Normal Distribuidas según su Capacidad de Adherencia a Células HEp-2

ADHERENCIA LOCALIZADA

\begin{tabular}{lcccc}
\hline & \multicolumn{2}{c}{ Positiva } & \multicolumn{2}{c}{ Negativa } \\
\hline & Diarreas & Asintomáticos & Diarreas & Asintomáticos \\
\hline $\begin{array}{l}\text { E. coli } 0111 \\
\begin{array}{l}\text { E. coli no entero- } \\
\text { patógeno. }\end{array}\end{array}$ & $11 / 13$ & $2 / 2$ & $5 / 7$ & $3 / 4$ \\
\hline
\end{tabular}




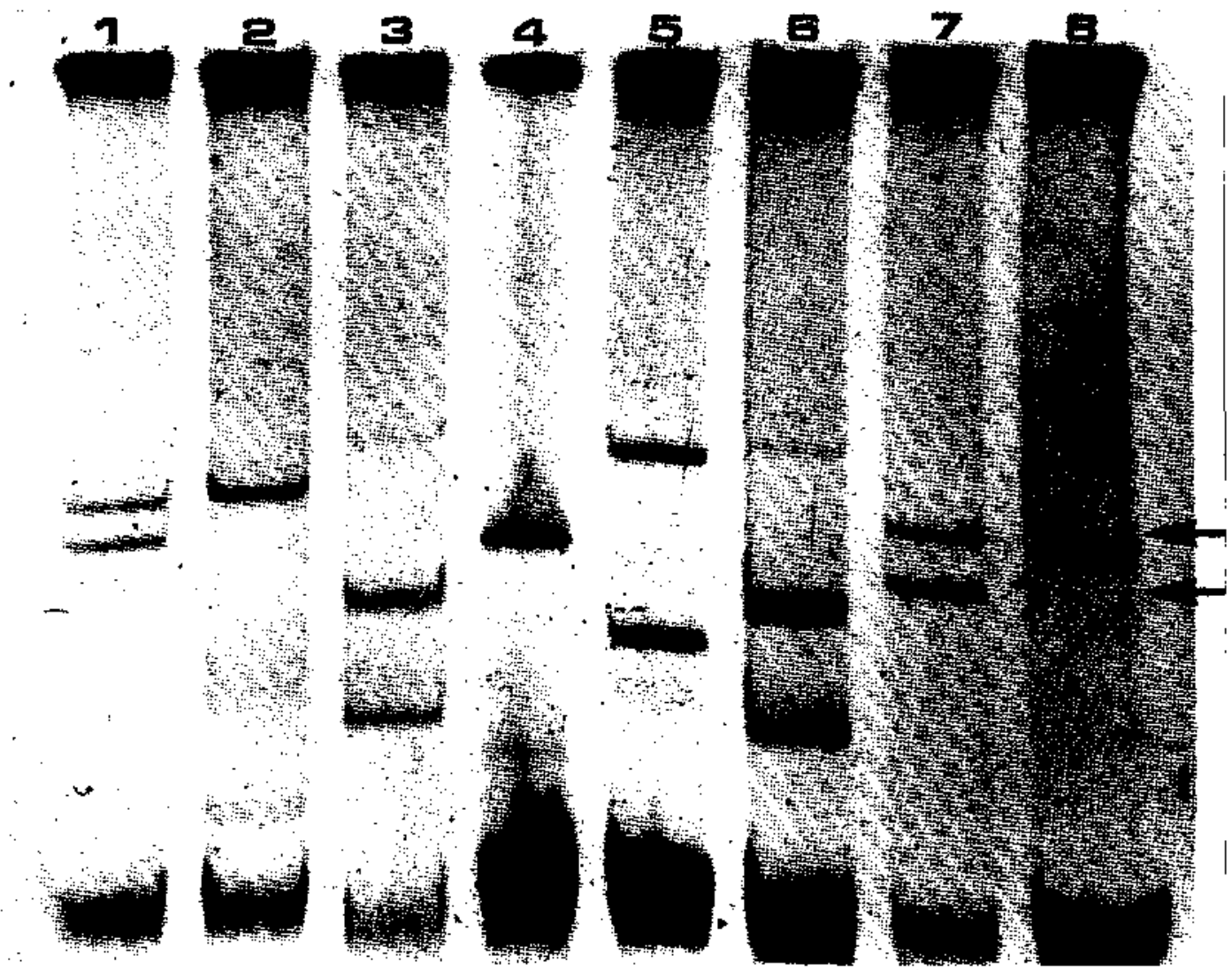

Fig. 3: Electroforesis en gel de agarosa. Fila 1 y $2 \mathrm{E}$, coli 0111 no adherente ( $68 \mathrm{Md}),(68 \cdots 50 \mathrm{Md})$; Fila 3 0111 adherencia localizada ( $105-47-28,1<26 \mathrm{Md})$; Fila 4 y 5 plasmidios de referencia: Plac $(101$ Md) y RP4 (36 Md), Rl (6l,Md) y SA (26 Md): Fila 6 E. coli 0111 adherencia localizada $(105-47-28-1<$ $26 \mathrm{Md}$ ): Fila 7 y $8 \mathrm{E}$. coli flora normal no adherente $(86 \cdot 1<26 \mathrm{Md}),(82 \cdot 65-2<26 \mathrm{Md})$.

Nota: Las flechas indican los plasmidios cuyos pesos moleculares varian entre 50 y $70 \mathrm{Md}$.

foresis de DNA plasmidial en cepas de E. coli 0111 con adherencia localizada y no adherentes y de E. coli de la flora norma] no adherentes.

\section{DISCUSION}

Los patrones de adherencia obtenidos en este estudio, en cepas de E. coli 0111, muestran menor frecuencia de aparición de adherencia localizada que en otras publicaciones 22,27 .

Las diferencias, podrían provenir de la selección de las muestras: 28 de las 33 cepas estudiadas por nosotros, correspondian al serogrupo 0.11. lo que implica incluir algunos serotipos que otros autores han excluido a priori. Probablemente ésta no sea la única explicación, ya que al analizar el comportamiento de las cepas consideradas como seratipos patógenos en otros estudios, las diferencias persisten, es asi como entre 5 cepas de algunos de los serotipos 0111:H2, 0111:H10, 0111:H12 y 0111: $\mathbf{H}^{-}$, sôlo en una se comprobó capacidad para desarrollar adherencia localizada (datos no mostrados). Aunque el número de cepas estudiadas fue pequeño, esta no pareciera ser la razón fundamental de las diferencias descritas. Posiblemente variaciones en el biotipo ${ }^{27}$ o de naturaleza ecológica, o bien la no improbable pérdida del plasmidio asociado a la capacidad de adherencia, pudiesen ser también explicaciones plausibles.

En cualquier caso es de gran interés estudiar mayor cantidad de cepas en nuestro medio, porque si estos datos se confirmaran, podría ponerse en duda la utilidad práctica de la serotipificación como único método de detección de ECEP.

En los resultados contenidos con las cepas de E. coli 0111 provenientes de niños asintomáticos. llama la atención que $50 \%$ de ellas desarrollan adherencia localizada, esto reforzaria estudios anteriores de nuestro laboratorio, que demuestran altas tasas de portación de este agente en niños menores de dos años ${ }^{28,29}$. Para de teminar la real perspectiva de estos hallazgos, sería necesario compararlos con estudios semejantes en otras áreas geográficas, desafortunadamente la revisión bibliográfica en esta materia hace pensar que tales informes no se encuentran disponibles. 
E] hallazgo de una entre catorce cepas de $\mathbf{E}$. coli de la flora "nomal" con capacidad de adherencia localizada, es algo más frecuente que lo informado por Nataro 22 : la trascendencia de este fenómeno, considerando el pequeño número de cepas estudiadas, requiere mayor investigación y puede obedecer al azar.

Nuestro intento de correlacionar la presencia de plasmidios con el fenómeno de adherencia resultó infructuoso, la razón principal fue la gran abundancia de plasmidios en el rango de PM 50 a $70 \mathrm{Md}$ y de plasmidios de otros pesos molecula. res en las cepas provenientes de niños sanos como enfermos. Estos resultados estimulan el interés por la posibilidad de utilizar en el futuro sondas genéticas, como método rápido y confiable para detectar la capacidad de adherencia de cepas ECEP.

\section{RESUMEN}

Mediante el ensayo in vitro con células $\mathrm{HEp}-2$, se evaluó la capacidad de adherencia de 33 cepas enteropatogénicas de E. coli 0111. Asimismo, se caracterizó en ellas la presencia de DNA plasmidial: 25 cepas provenían de lactantes con diarrea y 8 de pacientes asintomaticos. Se incluyeron como controles 14 cepas de $\mathbf{E}$. coli de la flora normal. No se encontraton diferencias en la capacidad adthesiva in vitro de las cepas de $\mathrm{E}$. coli 0111 aisladas de casos de diarrea $(60 \%)$ o de asintomáticos $(50 \%)$. En contrastre, en ambos grupos de cepas la frecuencia en que ocurrió la capacidad de adherencia fue significativamente mayor que la del grupo control $(7 \%) .(p<0,001$, prueba exacta de $F$ isher). La presencia de plasmidios con PM entre 50 y 70 Md fue demostrada tanto en las cepas que adherían como en las que no lo hacian. El ensayo de adherencia a células HEp-2 constituye una valiosa herramienta que permitirá mejorar el diagnóstico etiológico de la diarrea aguda.

\section{REFERENCLAS}

1. Ewing, W.H., Davis, B.R. Montagne, T.S.: Studies on the ocurrence of Escherichis coli serotypes associated with diarrheal disease. Center for Disease Control Atlanta, G.A. 1963.

2. Kauffmonn, F. Orskow, F.: Die bakteriologic der Escherichia coli - Enteritis, p. 1-4I. In A.A. am. (Ed.), Sauglingsenteritis. Georg Thieme Verlag, Stuttgart, 1956.

3. Edelman, R. Levine, M.M.: Summary of a workshop on enteropathogenic Escherichia coli. J. Infect. Dis. 147: $1108,1983$.

4. Dupant, H.L., Formal, S.B., Hornick, R.B., Synder, M.J., Libonati, J.P.; Pathoge nesis of Escherichia coli diarrhea, N. Engl. J. Med. 285: 1, 1971.

5. Myron, M., Levine, Robert Edelman: Enteropatho- genic Escherichia coli of classic serotypes associated with infant diarthea: Epidemiology and pathogenesis. Epidemiologic Reviews. 6: 31, 1984.

6. Sack, R.B.: Human diatrheal disease caused by enterotoxigenic Escheríchia coli. Ann Rev. Microbiol. 29: 333, 1975.

7. John, M. Deboy, Grace $M$. Thomas, Carl, F. Deneke: I. Kaye Wachsmuth. Colonization factors and Escherichia colj belonging to enterotoxin Associated serotypes, Curr. Microbiol. 6: 49, 1981.

8. Toledo, F.R.M. Reis, L.M.H., Almeida, G.R. Trabulsi, L.: Inyasive strains of Escherichia coli belonging to 0 group 29. J. Clin. Microbiol. 5: 288, 1979.

9. OBrien, A.D., Leveck, G.D., Thompson, M.R., Formal, S.B.: Production of Shigella dysenteriac 1 like cytotoxin by Escherichia coli. J. Infect. Dis. 146: 763,1982 .

10. Johnson, WM., I. for, H., Bezanson, G.S.: Cytotoxic Fscherichia coli 0157: 147 associated with haemorragic colitis in Canada. Lancet 14: ?6, 1983.

11. O'Brien, A.D., Levely, T.A., Chen, M.E., Rotman. $S$. W. Formal, S.B.: Escherichia coli 0157:H7 strains associates with haemorrhagic colitis in the United States produce a Shigella dy senteriac 1 (Shiga) like cytotox in. Lancet, March, 26: 702, 1983.

12. Levine, M.M., Bergquist, E.J., Nolin, D.R., Waterman, D.H., Horniex, R.B., Young, L.R.. Stomon, S.: Escherichia coli strains that cause diarthoeae but do not produce Heat-Labile or Heat-stable enterotoxins and are non invasive. Lancet 1: $1119,1978$.

13. Cravioto, A., Gross, R.J., Scotland, S.M. Rowe, B.: An adhesive factor found in strains of Escherichia coli belonging to the traditional infantile enteropathogenic serotypes. Curr. Microbiol. 3: 9S, 1979.

14. Scotland, S.M., Richmond, J.E, Rowe, B.: Adhesion of enteropathogenic strains of $E$ scherichia coli (EPEC) to HEp-2 cells is not dependent on the presence of fimbrias. FlimS Microbiol. Lett. 20 : $191,1983$.

15. Ulshen, M.H., Rollo, J.L.: Pathogenesis of Escherichia coli gastroenteritis in man-another mechaniss. N. Engl. J. Med, 302: 99, 1980.

16. Moon, H.W., Whipp, S.C., Argenzio, R.A., Levine, M.M.. Gionello, R.A.: Attaching and effacing activities of rabbit and human enteropathogenic Escherichia coli in pig and rabbit intestines. Infect: Immun, 41: 1340, 1983.

17. Contey Raberis, Willam, B. Lushbaugh, Lindsay, $R$. Inman.: Attachment of bacteria to intestinal epithelial cells in diarrhea caused by Escherichia coli strains RDEC-1 in the rabbit: Stage and role of capsule. J. linfect. Dis. 143, 219, 1981.

18. McNeish, A.S., Turner. D., Fleming, J. and Evans, $N$.: Mucosal adherence of human enteropathogenic Escherichia coli. Lancet 2: $946,1975$.

19. Scaletsky, I.C., Silvia, M.L. Trabulsi, L.: Distinctive patterns of adherence of enteropathogenic Escherichia coli to Hela cells. Inf. Imm. 48: 534, 1984.

20. Nataro. J.P., Scaletsky. I.C., Kaper, J.B., Levine, M.M. Trabusi, L.R.: Plasmid mediated factor: conferring diffuse and localized adherence of enteropathogenic Escherichia coli. Inf. Imm 48: $378,1985$.

21, Baldini, M.M., Kaper, J.B., Levine, M.M., Candy, D.C.A.; Moon. H.W.: Plasmid mediated adhesion in enteropathogenic Escherichia coli. J. Pediatr. Gastroenterol. Nutr. 3: 534, 1983. 
22. Natoro, J.P., Balaint, M.M., Kaper, J.B., Black, R.E., Bravo, N. Levine, M.M.: Detection of an adherence factor of Enteropathogenic Escherich in coli with a DNA probe. J. Infect. Dis, 152: 560, 1985.

23. Figueroa, G., Araya, M., Ibähez, S., Cler, $N$, Brunser, $O .:$ Enteropathogens associated acute diarrhea in hospitalized infants. J. Pediats. Gastroenterol. Nutr. 5, (en prensa), 1986.

24. Prado, V., Broun, S., Bosch, P., Bercovich, $M$. Reyes, C., Sawada, T.: Análisis de Escherichia coli enteropatógena clásica (ECEP) como una causa endémica de diarrea aguda en niños chilenos. Rev. Chil. Ped, 55: 171, 1984.

25. Kado, C., Liu, S.: Rapid procedure for detection and isolation of large and small plasmids. J, Bactetiol. 145: 1365, 1981.

26. Meyers, I.A., Sánchez, D.. Elwell, L.P., Falkow, S.:
Simple agarose gel electrophoretic method for the identification and characterization of plasmid deoxyribonucleic acid. J. Bacteriol. 127: 1529 , 1976.

27. Scaletsky, I.C., Situg, M.L., Toledo, M.R., Davis, B.R., Blake, P.A. and Trabulsi, L.R.: Correlation between adherence to Hela cells and serogroups. Serotypes, and bioserotypes of Eschetichia coli. Infect. Inm. 49:528, 1985.

28. Figueroa, G.: Estudio de prevalencia de serotipos enteropatógenos de Escherichia coli de deposiciones de nifíos que habitan una población suburbana de Santiago. Rev. Chil. Pediatr. 51: 225, 1980.

29. Figueroa, G., Troncoso, M., Araya. M., Espinoza, $J$. and Brunser, O.: Enteropathogen carriage by healthy individuals living in an area with poor sanitation. J. Hyg. Camb. 91: 499, 1983. 